\title{
Evaluation of Chewing and Swallowing Sensors for Monitoring Ingestive Behavior
}

\author{
Juan M. Fontana* and Edward S. Sazonov \\ Department of Electrical and Computer Engineering, The University of Alabama, 101 Houser Hall, \\ Tuscaloosa, AL, 35487-0286, USA
}

(Received: 21 November 2012. Accepted: 8 March 2013)

\begin{abstract}
Monitoring Ingestive Behavior (MIB) of individuals is of special importance to identify and treat eating patterns associated with obesity and eating disorders. Current methods for MIB require subjects reporting every meal consumed, which is burdensome and tend to increase the reporting bias over time. This study presents an evaluation of the burden imposed by two wearable sensors for MIB during unrestricted food intake: a strain sensor to detect chewing events and a throat microphone to detect swallowing sounds. A total of 30 healthy subjects with various levels of adiposity participated in experiments involving the consumption of four meals in four different visits. A questionnaire was handled to subjects at the end of the last visit to evaluate the sensors burden in terms of the comfort levels experienced. Results showed that sensors presented high comfort levels as subjects indicated that the way they ate their meal was not considerably affected by the presence of the sensors. A statistical analysis showed that chewing sensor presented significantly higher comfort levels than the swallowing sensor. The outcomes of this study confirmed the suitability of the chewing and swallowing sensors for MIB and highlighted important aspects of comfort that should be addressed to obtain acceptable and less burdensome wearable sensors for MIB.
\end{abstract}

Keywords: Chewing, Comfort Rating Scales, Eating Disorders, Monitoring Ingestive Behavior, Swallowing, Wearable Sensors.

\section{INTRODUCTION}

Wearable technology presents opportunities for long-term monitoring of individuals in the home and in community settings. ${ }^{1}$ Improvement in diagnosis and treatment can be achieved with wearable devices that not only focus on reliable design and functionality but also consider obtaining a high level of comfort for the wearer as these devices should be non-invasive and unobtrusive.

Monitoring Ingestive Behavior (MIB) is of importance to the study of eating behaviors in populations suffering from obesity and eating disorders. However, efficient MIB has been problematic due to current methods rely on subjects self-reporting their daily intake, which is timeconsuming, burdensome and may cause an increase in the reporting bias over time. ${ }^{2}$ The use of wearable devices has been proposed for MIB starting in laboratory studies and aiming to free living conditions. ${ }^{3,4}$ Such devices were introduced to overcome inaccurate self-reporting methods

\footnotetext{
${ }^{*}$ Corresponding author; E-mail: jmfontana@bama.ua.edu
}

and to lessen the reporting burden by objectively characterizing food intake episodes (occurrence, duration, rate of ingestion) and measuring the amount of food intake. ${ }^{5}$ while reducing the subject's participation on reporting the foods consumed. Several studies have reported the development of wearable devices for MIB,$^{5-7}$ however their main focus was the device functionality thus a comprehensive sensor burden analysis was not presented.

The burden imposed by wearable sensors can be evaluated by rating the level of comfort experienced by subjects when they perform different activities. The outcomes of this comfort analysis offer a suitable understanding of the burden and acceptability of the sensors under evaluation. A wide selection of tools have been presented for objective and subjective measurement of comfort in clinical settings and specialized areas. ${ }^{8}$ Postural comfort, thermal comfort, muscular fatigue and pain are some of the variables used to measure comfort levels. The limitation of studies using one of these variables lies in the fact that comfort should be analyzed taking into account multiple variables. Physical factors (weight and size of the device, 
how movement is affected, etc.) and psychological factors (appearance, embarrassment, etc.) may affect the levels of comfort observed by subjects suggesting that the wearable comfort should be assessed along several physical and cognitive dimensions. ${ }^{9}$ To address that problem and to determine what factors directly affect comfort, Knight and Baber developed a tool to measure the comfort of wearable computers. ${ }^{9}$ The proposed tool consists of a set of Comfort Rating Scales (CRS) that measured comfort across six different categories: Emotion, Attachment, Harm, Perceived Change, Movement and Anxiety. Each category defined a homogeneous aspect of comfort and grouped a wide variety of comfort terms related to wearing an object on the body. The principal advantage of using this tool is the ability to measure physical and physiological aspects of comfort, which are critical when evaluating the comfort and acceptability of wearable technologies. The CRS have been used to evaluate the comfort of a number of wearable devices and in different situations, ${ }^{10-14}$ and that can also be used to evaluate the burden of wearable sensors for MIB.

Wearable sensors for MIB should be unobtrusive, comfortable, and have a minimal impact on the way people eat. If some of these requirements are not fulfilled, subjects are less likely to use the sensors. Moreover, if the eating patterns of subjects are significantly affected by the presence of the sensors, then the benefits of using such sensors may be greatly reduced. Seeking for an unobtrusive and comfortable system for MIB, our research group is working on the development of a wearable monitoring device that integrates non-invasive sensors and pattern recognition methods for detection and characterization of food intake in a free living environment. ${ }^{3,5,15-17}$ The use of non-invasive sensors to accurately capture chewing and swallowing events was presented in a previous study. ${ }^{3}$ Information from chews and swallows was used to create models that detected food intake with more that 95\% accuracy. ${ }^{5}$ Functionality of chewing and swallowing sensors for MIB was further evaluated in different studies. ${ }^{15,16,18}$ In all of these, the sensors were placed in strategic locations on the body (immediately below the earlobe and above the gonial region of the mandible for the chewing sensor and over the laryngopharynx for the swallowing sensor) to capture relevant information while attempting to satisfy comfort requirements although a detailed comfort analysis was not reported.

The purpose of this study was to evaluate the burden introduced by chewing and swallowing sensors during experiments involving food intake. The chewing sensor consisted of a piezoelectric film strain sensor to detect jaw movements and the swallowing sensor consisted of a throat microphone to detect swallowing sounds. The sensors burden was evaluated by asking the subjects to rate their level of comfort after consuming four meals under unrestricted conditions. A questionnaire based on the comfort assessment tool developed by Knight and Baber ${ }^{9}$ was used to rate the level of comfort of subjects along several comfort dimensions. The outcomes of this study are being taken into consideration to further improve the design of the wearable monitoring device that is envisioned to objectively and accurately identify and characterize patterns of food intake while being unobtrusive and noninvasive.

This paper is organized as follows: Section 2 presents a general description of the experiments performed and a detailed description of the sensors used for MIB. Section 2 also includes the rationale of the methodology used to evaluate sensors burden. The results of such evaluation are presented in Section 3 along with a statistical analysis. Discussions and Conclusions are presented in Sections 4 and 5 respectively.

\section{METHODOLOGY}

\subsection{Data Collection}

A total of 30 healthy subjects (15 females and 15 males, average age $29.0 \mathrm{y} \pm 12.2 \mathrm{y}$ ) were recruited to participate in the data collection experiments. The study was approved by an Institutional Review Board at Clarkson University, Potsdam, NY and all subjects read and signed an informed consent form before participation. To evaluate the burden of the wearable sensors for various levels of adiposity, subjects in a wide range of BMI were recruited (average BMI $27.87 \mathrm{~kg} / \mathrm{m}^{2} \pm 5.51 \mathrm{~kg} / \mathrm{m}^{2}$ ).

Each subject completed a total of 4 visits, all of which were exactly at the same time of different days. Each visit consisted of three parts:

(1) a resting period in which subjects were asked to remain seated in a relaxed position for $5 \mathrm{~min}$;

(2) a meal period in which subjects had unlimited time to eat a meal of self-selected content and size; and

(3) a second resting period were subjects remained seated for $5 \mathrm{~min}$.

Before starting each experiment, volunteers were instrumented with a sensor system for monitoring ingestive behavior. ${ }^{19}$ The purpose of these sensors was to capture chewing and swallowing events while being non-invasive, unobtrusive and socially acceptable. Chewing and swallowing sensors were totally independent of each other in terms of how and where they were attached to the body.

\subsection{Chewing Sensor}

The sensor used to capture chewing events was a piezoelectric film strain sensor placed immediately below the earlobe, above the gonial region and over the posterior border of the ramus of the mandible as shown in Figure 1. This strain sensor detected characteristic jaw motion by monitoring changes in the skin curvature produced during chewing. Such skin curvature changes were caused by changes in the distance between the jaw and the temporal bones of the skull. ${ }^{16}$ 


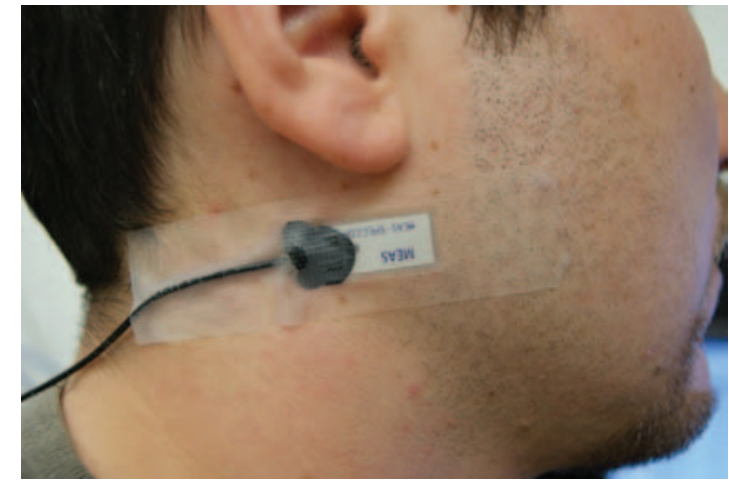

Fig. 1. Chewing sensor: a piezoelectric film strain sensor was attached immediately below the earlobe and above the gonial region of the mandible using medical tape.

The sensor selected was the LDT0-028K manufactured by Measurement Specialties (www.meas-spec.com). This sensor consists of a piezoelectric film element of $28 \mu \mathrm{m}$ thickness laminated to a $125 \mu \mathrm{m}$ polyester substrate resulting in a $0.153 \mathrm{~mm}$ thick, $25 \mathrm{~mm}$ long and $13 \mathrm{~mm}$ wide laminated sensor weighing less than $10 \mathrm{~g}$. Medical tape was used to attach the sensor to the skin, which intended to satisfy the requirements of non-invasiveness and unobtrusiveness.

\subsection{Swallowing Sensor}

The sensor used to capture swallowing events was a miniature throat microphone (IASUS NT) placed over the laryngopharynx (Fig. 2). This sensor detected characteristic sounds of swallowing originated when the bolus of food passes through the pharynx. ${ }^{3}$

To make the swallowing sensor wearable, the throat microphone was glued to a neoprene collar (paintball neck protector from JT Sports) that was designed to maintain a dry skin for a comfortable wear and breathability. The size

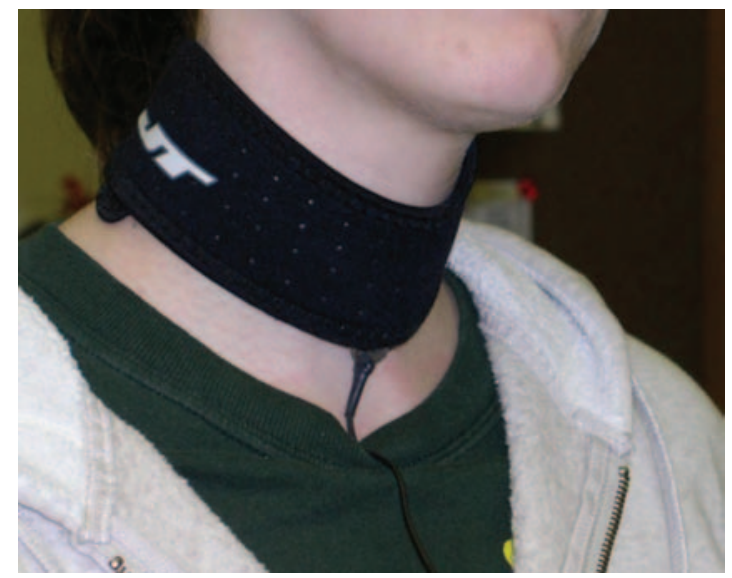

Fig. 2. Swallowing sensor: a miniature throat microphone was glued to a neoprene collar that was fastened to the neck of the subject using flexible velcro. Microphone was located over the laryngopharynx. of the selected collar was approximately $5 \mathrm{~cm}$ height and $47 \mathrm{~cm}$ long (55 cm long when it is fully stretched). These dimensions were sufficient to fit the wide variety of neck diameters of the subjects participating in the study. The weight of the collar with the throat microphone was less that $50 \mathrm{~g}$. Flexible Velcro was used to fasten the collar to the neck of the subject, thigh enough to capture suitable swallowing sounds while avoiding an unpleasant choking condition for the subject.

\subsection{Comfort Rating Scales (CRS)}

A questionnaire was designed based on the CRS developed by Knight and Baber ${ }^{9}$ to evaluate the burden of the chewing and swallowing sensors. The CRS were developed to measure comfort across six different categories called dimensions: Emotion, Attachment, Harm, Perceived Change, Movement and Anxiety. ${ }^{9}$ Emotion and Anxiety dimensions are associated to a psychological perception of comfort, whereas the remaining dimensions are associated to a physical perception of comfort. A rating scale was assigned to each comfort dimension to score the level of agreement of the subjects with a set of statements created based on the interpretation of each comfort dimension. ${ }^{9}$

The questionnaire designed for this study presented a set of 8 comfort statements, which were formulated to assess the comfort of the chewing and swallowing sensors (Table I). For the Movement dimension, two different statements were formulated to address both the effect of the sensors on body movement and the effect of the sensors on the way subjects ate their meals. A new dimension (Sensor Size) was added to address the benefits of a potential miniaturization of each sensor. All statements were addressed from a positive perspective meaning that the higher the score for a specific dimension, the higher the comfort and, consequently, the lower the burden of the sensor.

At the end of the last visit, subjects were asked to score their level of agreement with each one of the comfort statements presented in Table I. The scores ranged from 0 (lowest agreement) to 10 (highest agreement). Each sensor

Table I. Comfort statements created for each dimension of the comfort rating scale.

\begin{tabular}{|c|c|}
\hline Dimension & Definition \\
\hline Emotion & It is acceptable how I look when I wear the sensor. \\
\hline Attachment & $\begin{array}{l}\text { The sensor is well attached to my body. } \\
\text { I do not feel it moving. }\end{array}$ \\
\hline Harm & The sensor does not cause some pain and/or tickling \\
\hline Perceived change & I do not feel awkward wearing the sensor. \\
\hline Movement I & The sensor does not affect the way I move. \\
\hline Movement II & The sensor does not restrict the way I eat. \\
\hline Anxiety & I feel secure wearing the sensor. \\
\hline Sensor size & $\begin{array}{l}\text { A sensor of smaller size would increase } \\
\text { my level of acceptability. }\end{array}$ \\
\hline
\end{tabular}


was evaluated independently, so two scores were received for each comfort dimension, one for the chewing sensor and another for the swallowing sensor. Subjects were not aware of the presence of the comfort questionnaire until it was handled to them, in that way we attempted to keep the subjects focused mostly on eating their meal and not in the sensor comfort.

Two-tailed $t$-tests were performed to obtain statistically significant results for each dimension. The null hypothesis was that the scores for the chewing and swallowing sensors at a specific comfort dimension had the same mean. A $95 \%$ significance level was selected to determine whether the null hypothesis is rejected or not.

\section{RESULTS}

The scores provided by the subjects for each comfort dimension and for each sensor were averaged across all subjects and the results are presented in Figure 3. Anxiety was the comfort dimension with the highest scores for both sensors $(9.17 \pm 1.46$ for chewing sensor and $8.17 \pm 2.48$ for swallowing sensor) whereas Sensor size $(7.27 \pm 2.82)$ and Movement I $(5.47 \pm 3.13)$ were the dimensions with the lowest scores for the chewing and swallowing sensors respectively. Results of the statistical analysis showed that there were significant differences between the means of the scores at all comfort dimensions except at the Sensor Size dimension.

Sensor comfort was also compared for subjects with various levels of adiposity. For that purpose, the 30 subjects were grouped according to their BMI resulting in 11 obese $\left(\mathrm{BMI}>30 \mathrm{~kg} / \mathrm{m}^{2}\right), 10$ overweight (BMI between 25 and $30 \mathrm{~kg} / \mathrm{m}^{2}$ ) and 9 normal weight (BMI $<25 \mathrm{~kg} / \mathrm{m}^{2}$ ) subjects. Mean and standard deviations of the scores for each comfort dimension are presented in Table II along with $p$-values resulted from the statistical analysis.

\section{DISCUSSION}

This paper presented an evaluation of the burden imposed by two wearable sensors developed to monitor the ingestive behavior of individuals: a chewing sensor and a swallowing sensor. Sensors burden during food intake experiments was assessed by means of a questionnaire created based on the CRS. ${ }^{9}$ This questionnaire allowed the subjects to score their level comfort in different dimensions related to the physiological and physical perception of comfort. Each comfort dimension was defined by a statement that attempted to address a positive view towards the sensor comfort (Table I). Therefore, the higher the score received for each statement, the better the comfort perception experienced by the subjects and, thus, the lower the sensor burden. The analysis of the scores gathered from 30 subjects indicated that they presented high levels of comfort for both sensors. A comparison between the scores obtained for each sensor showed that the mean of the scores for the chewing sensor was higher than the mean of the scores for the swallowing sensor at all comfort dimensions suggesting that subjects felt more comfortable wearing the chewing sensor than the swallowing sensor. This result is supported by the statistically significant differences found between the scores received for each comfort dimension (Fig. 3).

The comfort dimensions analyzed in this study covered a wide range of factors related to the physical and physiological perception of comfort. For sensors developed for MIB, Movement is one of the most important dimensions to analyze since it is directly related to the level of obtrusiveness of the sensor during the eating period. It was critical to have high scores for this particular dimension as it would indicate that the sensors did not considerably affect the manner subjects ate and, therefore, they would not drastically change the subject's eating behavior. Movement was represented by two statements: Movement I addressing

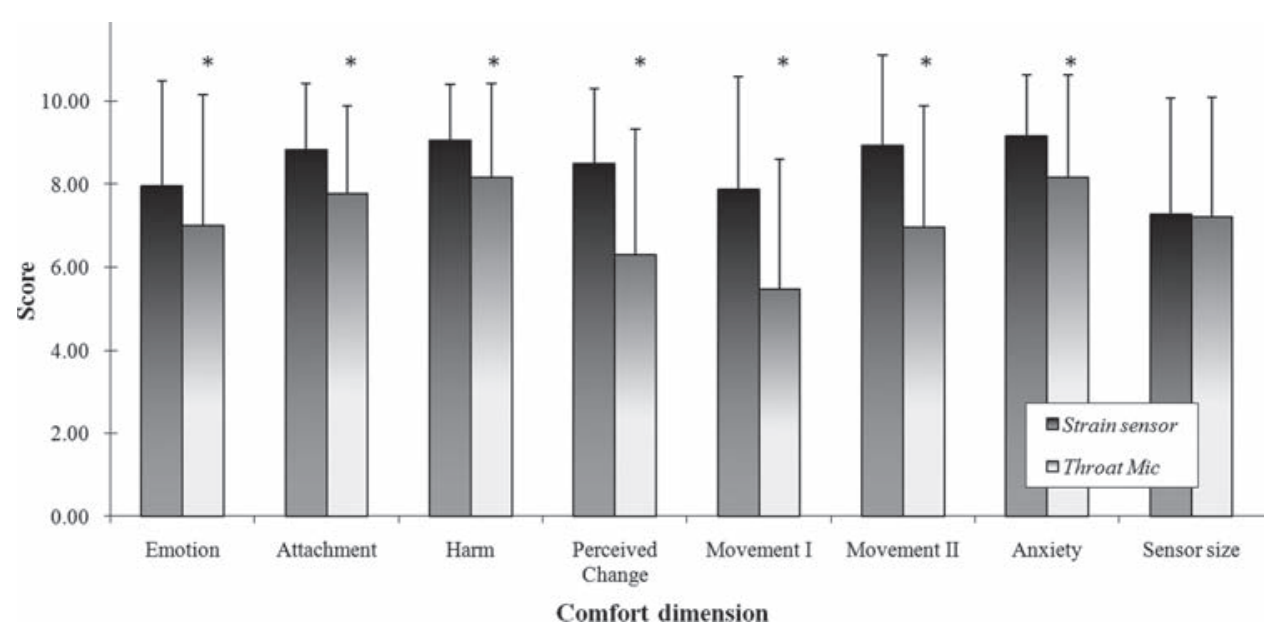

Fig. 3. Comfort assessment results for chewing and swallowing sensors used for monitoring ingestive behavior. Stars indicate that a statistically significant difference was observed between means. 
Table II. Statistical results of the comfort rating scales for normal weight, overweight and obese subjects.

\begin{tabular}{|c|c|c|c|c|c|c|c|c|c|}
\hline \multirow[b]{2}{*}{$\begin{array}{l}\text { Comfort } \\
\text { dimension }\end{array}$} & \multicolumn{3}{|c|}{ Normal weight } & \multicolumn{3}{|c|}{ Overweight } & \multicolumn{3}{|c|}{ Obese } \\
\hline & $\begin{array}{l}\text { Chewing } \\
\text { sensor }\end{array}$ & $\begin{array}{c}\text { Swallowing } \\
\text { sensor }\end{array}$ & $p$-value & $\begin{array}{c}\text { Chewing } \\
\text { sensor }\end{array}$ & $\begin{array}{c}\text { Swallowing } \\
\text { sensor }\end{array}$ & $p$-value & $\begin{array}{c}\text { Chewing } \\
\text { sensor }\end{array}$ & $\begin{array}{c}\text { Swallowing } \\
\text { sensor }\end{array}$ & $p$-value \\
\hline Emotion & $8.5 \pm 2.3$ & $8.0 \pm 2.6$ & 0.211 & $7.0 \pm 1.8$ & $5.3 \pm 3.2$ & 0.061 & $8.3 \pm 3.2$ & $7.4 \pm 3.4$ & 0.054 \\
\hline Attachment & $8.9 \pm 1.6$ & $8.0 \pm 2.2$ & 0.227 & $8.9 \pm 1.4$ & $7.3 \pm 2.2$ & 0.082 & $8.7 \pm 1.9$ & $7.9 \pm 2.1$ & 0.269 \\
\hline Harm & $9.5 \pm 0.9$ & $8.7 \pm 2.3$ & 0.285 & $9.1 \pm 1.1$ & $7.7 \pm 2.4$ & 0.021 & $8.5 \pm 1.5$ & $8.0 \pm 2.2$ & 0.513 \\
\hline Perceived change & $8.5 \pm 1.8$ & $6.5 \pm 2.9$ & 0.035 & $8.4 \pm 1.7$ & $5.4 \pm 3.0$ & 0.023 & $8.5 \pm 2.1$ & $6.8 \pm 3.3$ & 0.086 \\
\hline Movement I & $8.3 \pm 2.6$ & $5.5 \pm 3.2$ & 0.026 & $7.4 \pm 3.0$ & $4.9 \pm 3.0$ & 0.049 & $7.8 \pm 2.9$ & $6.0 \pm 3.4$ & 0.038 \\
\hline Movement II & $8.9 \pm 2.4$ & $7.5 \pm 2.7$ & 0.062 & $9.3 \pm 1.0$ & $6.1 \pm 3.2$ & 0.023 & $8.6 \pm 2.8$ & $7.1 \pm 3.0$ & 0.062 \\
\hline Anxiety & $9.6 \pm 0.9$ & $9.4 \pm 1.0$ & 0.192 & $8.8 \pm 1.6$ & $7.0 \pm 2.3$ & 0.091 & $9.0 \pm 1.8$ & $7.9 \pm 3.2$ & 0.162 \\
\hline Sensor size & $8.3 \pm 1.8$ & $7.7 \pm 2.0$ & 0.192 & $6.6 \pm 3.1$ & $7.3 \pm 3.3$ & 0.393 & $6.8 \pm 3.4$ & $6.5 \pm 3.5$ & 0.678 \\
\hline
\end{tabular}

the way subjects move and Movement II addressing the way subjects eat. Results of scoring Movement II dimension for the chewing sensor showed a very high mean value $(8.93 \pm 2.18)$ meaning that the obtrusiveness of this sensor was minimal and subjects would not significantly change the manner they eat when wearing the chewing sensor. On the other hand, the mean of the scores for the swallowing sensor in the same dimension was significantly lower than the mean of the chewing sensor scores, although its value $(6.97 \pm 2.93)$ indicated that subjects did not experience a critical discomfort during food intake but that further improvement of the sensor design is necessary to increase its acceptance. Similar results were observed for Movement I dimension with a significantly higher mean of scores for the chewing sensor. The scores mean for the swallowing sensor was slightly above the scale midpoint $(5.47 \pm 3.13)$ which points out that this is the main aspect of comfort that needs to be addressed for this sensor to reduce intrusion and improve comfort. Attachment was another important dimension to analyze due to sensors for MIB are intended to be worn for long periods of time $(\sim 24 \mathrm{~h})$, so their presence in the body should pass unnoticed for the subjects wearing them. The chewing sensor was well attached to the body and most of the subjects did not feel the sensor moving as revealed by an $8.83( \pm 1.60)$ score mean. However, the use of medical tape to attach the chewing sensor to the body may be a limitation due to several factors such as perspiration, beard or a moisturized skin may cause sensor detachment. Medical adhesive overcome those issues and it is currently being tested in laboratory experiments as an alternative solution.

Chewing sensor scores for Perceived Change and Harm were high $(>8.5)$ suggesting that the sensor is not causing niggling pain and it is not inconvenient to wear, therefore these aspects of comfort do not represent a major concern to be addressed. On the other hand, low scores in the Perceived Change dimension for the swallowing sensor suggested that this aspect should be addressed in future designs to improve comfort. Harm scored above 8 points for the same sensor indicating that it is not causing significant pain and that addressing this aspect of comfort is not a priority.
Sensor size was a new dimension included into the questionnaire to determine whether a reduction in the sensor size would improve the acceptability of the sensor. Mean score values for chewing and swallowing sensors were similar and a relative high level of agreement was observed, thus highlighting the importance of having smaller sensors to improve comfort.

Subjects indicated that the chewing sensor was perceived more 'psychologically' comfortable than the swallowing sensor, which was supported by the statistically significant differences found in the scores for Emotion and Anxiety dimensions. Emotion addressed appearance and relaxation concerns whereas Anxiety addressed the sense of worry and safety that subjects had when wearing the sensors. The maximum scores for each sensor across all dimensions were achieved in the Anxiety dimension, which clearly showed that subjects felt secure when wearing the sensors. Mean scores above 7 for both sensors in the Emotion dimension indicated no major concerns in subject's appearance when wearing the sensors although the design of wireless "band-aid" sensors may increase the scores for this comfort dimension.

Subjects were grouped according to their BMI and the mean scores for each comfort dimension was computed to gain a better understanding of the levels of comfort experienced by subjects on each group. Subjects belonging to the normal weigh group scored higher for the chewing sensor than for the swallowing sensor at all comfort dimensions. However, only the scores for Movement I and Perceived Change dimensions showed statistically significant differences. For the chewing sensor, the lower mean score was 8.3 for Movement I, suggesting that this sensor was predominantly comfortable for this group of people. Overweight subjects scored higher for the chewing sensor than for the swallowing sensor in 7 out of 8 dimensions. Statistically significant differences between scores for each sensor were found for Movement I, Movement II and Perceived Change dimensions. Emotion and Perceived Change were the dimensions with the lowest score means for the chewing and swallowing sensors respectively. Finally, the scores reported by obese subjects were higher for the chewing sensor than for the swallowing 
sensor at all comfort dimensions. Movement I scores presented statistically significant differences between sensors. For the chewing sensor, the lowest mean score $(6.8 \pm 3.4)$ was obtained in the Sensor Size dimension suggesting that obese subjects would not greatly benefit from a sensor miniaturization. For the swallowing sensor, Movement I $(6.0 \pm 3.4)$ and Perceived Change $(6.8 \pm 3.3)$ were the aspects of comfort representing a major concern for this group.

The results presented in this study reflect short term experiments (about 1 hour) which may constitute a limitation to fully understand the level of burden imposed by the sensors. However, as the experiments involved the consumption of four different meals in different days, the results allowed obtaining valuable information about the sensors burden during food intake episodes. Long-term studies $(\sim 24 \mathrm{hrs})$ are required to evaluate the sensors burden when subjects perform different activities of daily living. Additionally, functionality is another important factor that may be included in the sensors burden evaluation as it directly affects the comfort. ${ }^{20}$ For this study, functionality of the chewing and swallowing sensors was not evaluated since their reliability was previously established by our research group. ${ }^{16,18}$

Since the development of a low cost and socially accepted monitoring device may probably demand the use of only one sensor, the results achieved in this study across all comfort dimensions suggested that the chewing sensor may be more suitable for MIB, in terms of comfort, compared to the swallowing sensor.

\section{CONCLUSION}

This paper presented an assessment of the burden imposed by two different wearable sensors for monitoring ingestive behavior: a chewing sensor and a swallowing sensor. Thirty subjects wore the sensors when eating a full meal during 4 separated visits. After the last visit, subjects scored their level of agreement with different statements that addressed the perception of comfort in different dimensions. The most important dimension addressed the changes in the eating habits of the subjects. Results indicated that the way the subjects ate their meals was not significantly affected by the presence of the sensors. An statistical analysis showed that the mean of the chewing sensor scores was higher than the mean of the swallowing sensor scores in most of the comfort dimensions suggesting that participants felt more comfortable wearing the chewing sensor than the swallowing sensor. Outcomes of this study will be used to decide on the most suitable sensor approach and to improve current and future development of a wearable ingestion monitor.

Acknowledgments: The project described was partially supported by Grant Number R21DK085462 from the National Institute of Diabetes and Digestive and Kidney Diseases. The content is solely the responsibility of the authors and does not necessarily represent the official views of the National Institute of Diabetes and Digestive and Kidney Diseases or the National Institutes of Health.

\section{References and Notes}

1. P. Bonato, IEEE EMBS Magazine 29, 25 (2010).

2. A. H. C. Goris, E. P. Meijer, and K. R. Westerterp, Br. J. Nutr. 85,629 (2001)

3. E. Sazonov, S. Schuckers, P. Lopez-Meyer, O. Makeyev, N. Sazonova, E. L. Melanson, and M. Neuman, Physiol Meas. 29, 525 (2008)

4. S. Päßler, M. Wolff, and W.-J. Fischer, Physiol Meas. 33, 1073 (2012).

5. E. S. Sazonov, S. A. C. Schuckers, P. Lopez-Meyer, O. Makeyev, E. L. Melanson, M. R. Neuman, and J. O. Hill, Obesity 17, 1971 (2009).

6. O. Amft, Sensors, 2010 IEEE 222, (2010).

7. M. Sun, J. D. Fernstrom, W. Jia, S. A. Hackworth, N. Yao, Y. Li, C. Li, M. H. Fernstrom, and R. J. Sclabassi, J. Am. Diet Assoc. 110, 45 (2010)

8. E. J. M. Pearson, Disability and Rehabilitation: Assistive Technology 4, 301 (2009).

9. J. F. Knight and C. Baber, Human Factors: The Journal of the Human Factors and Ergonomics Society 47, 77 (2005).

10. J. F. Knight and C. Baber, Applied Ergonomics 38, 237 (2007).

11. W. J. Tharion, M. J. Buller, A. J. Karis, and S. P. Mullen, Proceedings of the Human Factors and Ergonomics Society Annual Meeting (2007), Vol. 51, p. 1006.

12. G. Cho, S. Lee, and J. Cho, International Journal of HumanComputer Interaction 25, 582 (2009).

13. P. Weller, L. Rakhmetova, Q. Ma, and G. Mandersloot, Pers Ubiquit Comp. 14, 73 (2010).

14. M. G. Tsipouras, A. T. Tzallas, G. Rigas, S. Tsouli, D. I. Fotiadis, and S. Konitsiotis, Artificial Intelligence in Medicine 55, 127 (2012).

15. P. Lopez-Meyer, O. Makeyev, S. Schuckers, E. Melanson, M. Neuman, and E. Sazonov, Ann. Biomed. Eng. 38, 2766 (2010).

16. E. S. Sazonov and J. M. Fontana, IEEE Sens J. 12, 1340 (2012).

17. O. Makeyev, P. Lopez-Meyer, S. Schuckers, W. Besio, and E. Sazonov, Biomedical Signal Processing and Control 7, 649 (2012).

18. E. Sazonov, O. Makeyev, P. Lopez-Meyer, S. Schuckers, E. Melanson, and M. Neuman, IEEE Trans. Biomed. Eng. 57, 626 (2010).

19. J. M. Fontana, P. Lopez-Meyer, and E. S. Sazonov, 2011 Annual International Conference of the IEEE Engineering in Medicine and Biology Society, EMBC (2011), p. 1884.

20. K. Bodine and F. Gemperle, Proceedings of the Seventh IEEE International Symposium on Wearable Computers (2005), p. 57. 